\title{
Gefälligkeitsvasektomie - nach wie vor ein umstrittenes Thema
}

\author{
V. Müller-Mattheis ${ }^{1}$, W. Bonte ${ }^{2}$, R. Ackermann ${ }^{1}$ \\ Urologische Klinik und Poliklinik der Universität Düsseldorf ${ }^{1}$ (Direktor: Prof. Dr. R. Ackermann) \\ Institut für Rechtsmedizin der Universität Düsseldorf ${ }^{2}$ (Direktor: Prof. Dr. W. Bonte)
}

In der Bundesrepublik Deutschland lassen sich jährlich schätzungsweise zwischen 35000 und 40000 Personen aus familienplanerischen Motiven sterilisieren. Seit einigen Jahrzehnten beschäftigen sich zahlreiche Publikationen der medizinischen und juristischen Literatur damit,

- ob Sterilisationseingriffe bei Mann und Frau strafrechtlich zulässig seien,

- ob es zivilrechtliche Bedenken gegen diese Art des Vorgehens gebe oder

- ob bei einer erfolglos durchgeführten Sterilisation der dafür verantwortliche Arzt Schadensersatz leisten müsse.

Es besteht nach wie vor eine große Rechtsunsicherheit, da in den veröffentlichten Urteilen und Rechtskommentaren widersprüchliche Rechtsauslegungen und Gesetzesinterpretationen zu finden sind.

Der in der 6. Legislaturperiode von der Bundesregierung eingebrachte Entwurf eines 5. Strafrechtgesetzes sah eine strafrechtliche Neuregelung von Schwangerschaftsabbruch und Sterilisation vor (veröffentlicht in der Bundestagsdrucksache VI/3434). Die Reform des Abtreibungsstrafrechtes erschien seinerzeit vordringlich einer Regelung zu bedürfen, so daß die weitere Bearbeitung des Entwurfes, soweit er die freiwillige Sterilisation betraf, zurückgestellt wurde. Nach Abschluß der Reformarbeiten zum Schwangerschaftsabbruch wurde er jedoch wieder aufgegriffen.

\section{Indikationsstellung}

Die Sterilisationseingriffe lassen sich in sechs Fallgruppen aufschlüsseln (Tab. 1).

Während bei den in der Tabelle aufgeführten Indikationsstellungen 1-5 die Rechtslage geklärt erscheint, ist dies bei der Sterilisation ohne Indikation (Gefälligkeitssterilisation) nicht der Fall.

\section{Tab. 1 Indikationsstellung zur Vasektomie}
1. medizinische Indikation
2. medizinisch-kriminologische und medizinisch-soziale Indikation
3. eugenische Indikation
4. vikariierende Indikation
5. soziale Indikation
6. Gefälligkeitssterilisation

\begin{abstract}
Die nachfolgenden Ausführungen sollen zum Verständnis der gegenwärtigen Rechtslage dienen.
\end{abstract}

Zur Begriffserklärung der Gefälligkeitssterilisation finden sich in der Literatur verschiedene Definitionen. So definiert Spann sie als „Sterilisation ohne ärztliche oder soziale Indikation“, Hörster sieht in ihr eine „Sterilisation, deren einzig mögliche, nicht weiter ableitbare Rechtfertigung in dem Wunsch der betreffenden Person liegt, jedes mit dem Geschlechtsverkehr verbundene Schwangerschaftsrisiko auszuschließen“, v. Massenbach spricht von „einer die Fortpflanzungsfähigkeit endgültig aufhebenden Operation zur Erleichterung des Sexuallebens oder der äußeren Lebensumstände“, oder es wird in Gerichtsurteilen von einer „Sterilisation ohne jeden nachvollziehbaren Grund“ gesprochen.

Zunehmend wird an den operativ tätigen Urologen von verheirateten und unverheiratenen Männern der Wunsch nach Gefälligkeitsvasektomie herangetragen. Die hier erörterten Aspekte sollen eine Hilfestellung bei der Entscheidung, ob er diese Operation durchführen darf oder nicht, geben.

Nach dem Reichsstrafgesetzbuch von 1871 waren bei jeder Unfruchtbarmachung von Mann und Frau Indikation oder Zielsetzung waren gleichgültig - die Tatbestände der Körperverletzung nach den $\$ \S 223,224$ und 225 StGB erfüllt. Es konnten bis zu zehn Jahre Zuchthausstrafe verhängt werden, wenn eine Körperverletzung den Verlust der Fortpflanzungsfähigkeit zur Folge hatte. Wenn sich der Arzt auf einen besonderen „Rechtfertigungs- oder Entschuldigungsgrund" berufen konnte, blieb diese Körperverletzung straflos. Ob die Sterilisierung lediglich Nebenfolge eines aus anderen Gründen indizierten Eingriffes war oder ob sie das erklärte Primärziel darstellte, war unerheblich: In jedem Fall wurde der Eingriff als Körperverletzung eingestuft und bedurfte einer besonderen Rechtfertigung. Diese war durch die Einwilligung des Patienten gegeben.

Obwohl man meinen könnte, daß damit bei den freiwilligen Sterilisationen das Problem gelöst worden wäre, war dem nicht so. Denn zu den juristischen Grundlehren der Einwilligung gehört die Voraussetzung, daß diese nur dann rechtswirksam ist, wenn der Einwilligende über das betroffene Rechtsgut verfügen kann. Dieses Verfügungsrecht stand dem Patienten höchstens bei therapeutischen Heileingriffen zu, es wurde ihm bei der freiwilligen Sterilisation abgesprochen. Die Ausnahme bildete die medizinische Indikation bei der Frau.

Akt. Urol. 19 (1988) 325-330

(C) Georg Thieme Verlag Stuttgart · New York 
Diese Rechtsprechung wurde noch im Mai 1933 durch ein Sterilisationsurteil des Reichsgerichtes bestätigt (RG, Juristische Wochenschrift 1933, 2060). Das „Gesetz zur Verhütung erbkranken Nachwuchses“ (Erbgesundheitsgesetz) trat am 1. Januar 1934 in Kraft. Mit Wirkung ab 1.6. 1933 war $\$ 226$ a in das StGB eingeführt worden (RGBl. I S. 295). Dieser Paragraph besagte, daß jede Art von Körperverletzung (Eingriff in die körperliche Integrität), selbst sogar schwere Körperverletzungen gerechtfertigt waren, wenn der Betroffene dieser Körperverletzung zugestimmt hatte. Eine Körperverletzung war nicht gerechtfertigt, wenn die Tat gegen die guten Sitten verstieß! Eine Sittenwidrigkeit wurde bei allen medizinisch indizierten Eingriffen verneint. Das Erbgesundheitsgesetz hatte die Frage nach der Strafbarkeit von Sterilisierungen abschließend so geregelt, daß es Sterilisierungen in im Gesetz näher bezeichneten Fällen zuließ (medizinische, eugenische, kriminologisch-sexualmedizinische Gründe); alle anderen Sterilisierungen waren ausnahmslos auch dann als schwere Körperverletzungen zu bestrafen, wenn sie mit Einwilligung des oder der Betroffenen vorgenommen worden waren. Die Rechtfertigung durch die Einwilligung entfiel hiernach für freiwillige Sterilisierungen schlechthin, und es kam daher auch nicht darauf an, ob die Tat gegen die guten Sitten verstieß oder nicht.

Diese Sonderregelung des Erbgesundheitsgesetzes wurde 1943 als $\$ 226$ b in das StGB eingeführt und dahingehend erweitert, daß die Unfruchtbarmachung, sofern sie nicht in den gesetzlich zugelassenen Fällen genehmigt war, auch dann strafbar sein sollte, wenn sie der Betreffende an sich selbst vornahm bzw. wenn jemand bei sich selbst oder bei einem anderen die Sterilisierung durch Bestrahlung oder Hormonbehandlung erreichte.

\$226b StGB ist durch Artikel I des Kontrollratsgesetzes Nr. 11 vom 30.1. 1946 über die Aufhebung einzelner Bestimmungen des deutschen Strafrechts ersatzlos gestrichen worden, denn man hat in ihm nach Beendigung des 2 . Weltkrieges noch nationalsozialistisches Gedankengut gesehen. Das Kontrollratsgesetz seinerseits wurde durch Artikel 2 des Gesetzes Nr. A-37 der Alliierten Hohen Kommission vom 5. 5. 1955 unwirksam gemacht. Das bedeutete allerdings nicht, daß der durch das Kontrollratsgesetz Nr. 11 aufgehobene $\$ 226$ b StGB wieder in Kraft getreten wäre! Aufgrund dieser beiden verzahnten Gesetze (Kontrollratsgesetz Nr. 11, Gesetz Nr. A-37 der Alliierten Hohen Kommission) trat eine brisante Rechtslage ein: Für die juristische Bewertung von Sterilisationen stand lediglich $\$ 226$ a StGB zur Verfügung. Nach dem Rechtsgedanken dieses Paragraphen war also bei einer freiwilligen Sterilisation zu prüfen, ob die Einwilligung in die Sterilisation als sittenwidrig anzusehen war oder nicht. War die Einwilligung gegen die guten Sitten gerichtet, so galt sie aufgrund dieser Tatsache als hinfällig!

Nun kam es zu einem spektakulären Fall in der Deutschen Rechts- bzw. rechtsmedizinischen Geschichte:

Das Landgericht Hannover verurteilte 1963 den Arzt Dr. Axel Dohrn wegen 149 freiwilliger Sterilisationen, die er zumeist aus sozialer Indikation vorgenommen hatte. Um nicht gezwungen zu sein, eine mehrjährige Zuchthausstrafe zu verhängen, die dem Gericht offenbar unangemessen erschien, nahm man neben $\S 226 \mathrm{a}$ StGB den $\S 216 \mathrm{StGB} \mathrm{z} \mathrm{u} \mathrm{Hilfe} \mathrm{und} \mathrm{erkannte} \mathrm{auf}$ eine Strafe von 6 Monaten Gefängnis. $\$ 216$ StGB (Tötung auf Verlangen) besagt, daß jemand, der durch das ausdrückliche und ernstliche Verlangen des Getöteten zur Tötung bestimmt worden ist, mit einer Freiheitsstrafe von 6 Monaten bis zu 5 Jahren bestraft wird. Dieses Urteil des Landgerichts Hannover ging in die Revision. Die Antwort des 5. Strafsenats in Berlin lautete: „Es gibt keine deutsche Strafvorschrift mehr, die eine freiwillige Sterilisation mit Strafe bedroht. Diese Gesetzeslücke kann nur durch den Gesetzgeber geschlossen werden.“ (BGHST. 20, 81 vom 27 10. 1964).

Die oben aufgeführte rechtsgeschichtliche Entwicklung führt zum Verständnis der Begründung dieses richtungweisenden Urteils des BGH. In der Begründung wird ausgeführt, daß durch die Aufhebung des $\$ 226 \mathrm{~b}$ durch Artikel I KRG Nr. 11 alle Körperverletzungstatbestände nicht mehr in Betracht kämen, soweit diesen durch die Einführung des \$226b im Jahre 1943 ihre Geltung genommen worden war. Andere als die im Erbgesundheitsgesetz zugelassenen Sterilisierungen stellte allein $\$ 226 \mathrm{~b}$ unter Strafe. $\$ 226$ a sei durch die abschließende Regelung des Erbgesundheitsgesetzes rechtlich bedeutungslos geworden, dem $\$ 226$ a sei im übrigen eine Lösung des weitschichtigen Sterilisierungsproblems nicht zu entnehmen. In der juristischen Fachwelt wurden Urteil des BGH und dessen Begründung heftig kritisiert.

Die Problematik bestand also darin, daß \$226b StGB, der speziell die freiwillige Sterilisation unter Strafe gestellt hatte, ersatzlos gestrichen war, es bestand gleichsam ein rechtsfreier Raum.

In einer ausführlichen Diskussion um die Strafwürdigkeit der Gefälligkeitssterilisation vertritt Hoerster die Meinung, daß unter dem Gesichtspunkt des Verstoßes gegen die Moral die Gefälligkeitssterilisation nicht strafwürdig sei. Es wird in diesen Ausführungen klar betont, daß „die guten Sitten“ bzw. „die richtige Moral“ praktisch nicht mit Allgemeingültigkeit zu definieren seien. Aber selbst wenn dies möglich wäre, erschiene es seiner Meinung nach nicht einsichtig, die Moral als solche zum Schutzobjekt des Strafrechtes zu machen. Schwieriger ist nach Ansicht Hoerster's die Betrachtungsweise der Gefälligkeitssterilisation als Schädigung. Der Eingriff in die körperliche Integrität und der dauerhafte Entzug der Fortpflanzungsfähigkeit stellen einen „Schaden“ dar, wie immer die Grenzen dieses Begriffs bestimmt seien. Ein Schaden sei nicht schon deshalb zu verneinen, weil der Betroffene in die Schädigung eingewilligt habe oder gar diesen Schaden in eigener Täterschaft herbeigeführt habe.

Ist nun eine solche Schädigung selbst dann als strafwürdig anzunehmen, wenn der Geschädigte in sie in wirksamer Weise eingewilligt hat? Hat der Staat also das Recht oder sogar die Pflicht, den erwachsenen Bürger auch vor solchen Schädigungen zu schützen, mit denen er selbst ausdrücklich einverstanden ist? Sollte der Staat den einzelnen, der in voller Kenntnis der Tragweite seiner Entscheidung in eine Schädigung einwilligt, tatsächlich so weitreichend bevormunden dürfen? Dem Staat fiele dann gleichsam die Aufgabe zu, den einzelnen vor sich selbst zu schützen. In seinen weiteren Ausführungen kommt Hoerster zu dem Schluß, daß das Strafrecht zwar nicht dazu existiere, die Moral zu schützen oder zu stärken, aber es darf sich nicht vor jedem noch so vorübergehenden Wunsch des einzelnen respektvoll zurückziehen. Hoerster gesteht der Strafrechtsordnung die Kompetenzen zu, den einzelnen immer dann vor Schädigungen durch andere zu bewahren, wenn dessen eigene dauerhafte Interessen dieses erfordern. 
Als Beispiel wird die Verstümmelung eines Menschen herangezogen. In aller Regel würde der Verstümmelte, was immer seine Motivation bei der Abgabe der Einwilligung war, ihr Ergebnis später bedauern. Grundsätzlich sieht Hoerster die Möglichkeit einer Bestrafung der Gefälligkeitssterilisation als gegeben an.

In diesem Zusammenhang darf eine andere wesentliche Publikation nicht außer acht gelassen werden: 1967 hat $T$. Wulfhorst in seiner Abhandlung ,Wäre eine Strafbarkeit der freiwilligen Strerilisierung verfassungswidrig?" klar zum Ausdruck gebracht, daß er dies für unvereinbar mit dem Grundgesetz hielte. In seiner Begründung stützt sich Wulfhorst auf Art. 6 des Grundgesetzes, der Ehe und Familie unter den besonderen Schutz der staatlichen Ordnung stellt. Dem einzelnen Bürger wird hierdurch ein Abwehrrecht gegen störende und schädigende Eingriffe des Staates in seine Ehe und seine Familie zuerkannt. Bei der Auslegung aller Grundrechte muß weiterhin die Würde des Menschen berủcksichtigt werden, die nach Art. 1 des Grundgesetzes unantastbar und von aller staatlichen Gewalt zu achten und zu schützen sei. Wulfhorst zieht unter anderem den Schluß, daß staatliche Vorschriften, die die Fortpflanzungsfähigkeit der im wesentlichen gesund erscheinenden Bürger regelt, also Bestimmungen über die Anzahl der zu zeugenden oder zu empfangenen Kinder, ein Verbot der Empfängnisverhütung und beschränkende Regelungen über einzelne empfängnisverhütend wirkende Mittel, die Würde des Menschen verletzen. Wenn der Staat seinen Bürgern nicht vorschreiben dürfe, ob, wann und wie oft sie sich fortpflanzen müßten, könne sich der einzelne gegen staatliche Reglementierungen der Familienplanung in Form des Verbotes der freiwilligen Sterilisierung wehren. Es wird weiterhin auf Art. 2 des Grundgesetzes hingewiesen, nach dem jeder das Recht auf die freie Entfaltung seiner Persönlichkeit hat, soweit er nicht die Rechte anderer verletzt und nicht gegen die verfassungsmäßige Ordnung oder das Sittengesetz verstößt. Nach der Rechtsprechung des Bundesverfassungsgerichtes wird die allgemeine Handlungsfreiheit durch Art. 2 GG geschützt.

Insofern sieht Wulfhorst keine Berechtigung des Staates, daß dieser ein Reglementierungsrecht über Verhütungsmethoden, die dem einzelnen nach seinen Bedürfnissen und seiner Ethik gemäß erscheinen, besitzt. Nach Wulfhorst's Ansicht kann die freiwillige Sterilisierung nicht einmal als eine Körperverletzung im Rechtssinne angesehen werden. Rein physiologisch betrachtet beinträchtigt sie zwar die Unversehrtheit des Körpers, schafft aber, funktionell aus der Sicht des Sterilisierten gesehen, keinen im Rechtssinn krankhaften Zustand, in dem eine lebenswichtige oder gar notwendige oder nur irgendwie wertvolle Körperfunktion fehlt. Die freiwillige Sterilisierung darf auch nicht als willkürliche Selbstverstümmelung gewertet werden, weil sie zu einem bestimmten, vom Sterilisierten autonom beherrschten Zweck, nämlich der Empfängnisregelung, vorgenommen werde. Sie sei außerdem qualitativ nicht anders einzuordnen als die hormonelle Steuerung, bei der die natürlichen Fortpflanzungsvorgänge ebenfalls tiefgreifend, wenn auch voraussichtlich nur vorübergehend, verändert würden.

In diesem Zusammenhang muß darauf hingewiesen werden, daß das Dohrn-Urteil eine Einzelfallentscheidung darstellt, der in keinem Fall Gesetzeskraft zukommt, sondern nur für diesen einen Prozeß Gültigkeit besitzt. Infolgedessen kann jedes andere deutsche Gericht in einem fachlich gleichliegenden Verfahren mit gleicher Verbindlichkeit anders entscheiden! Da auf der anderen Seite dem Musterspruch des BGH eine "faktische Gesetzeskraft" zukommt, kann man mit hoher Wahrscheinlichkeit davon ausgehen, daß sich Amts- und Landgerichte wie auch Staatsanwaltschaften an der höchstrichterlichen Entscheidung orientieren.

Wegen der Unmöglichkeit, den Begriff „Sittenwidrigkeit" zu objektivieren und der Freiheitsmaxime im intimsten persönlichen Lebensbereich, dessen Gestaltung nicht durch Kriminalstrafe geregelt werden darf, hat unter anderem auch Roxin 1964 (JuS 1964, 380) und 1965 (Niedersächsisches Ärzteblatt Nr. 6) eine nahezu unbeschränkte Freigabe freiwilliger Sterilisierungen gefordert.

Der Bundesgerichtshof hat 1976 (BGH, NJW 1976, 1790) erneut zu der umstrittenen Frage der Rechtmäßigkeit der freiwilligen Sterilisation Stellung genommen, di e s mal nicht in einer strafrechtlichen, sondern in einer zivilrechtlichen Entscheidung:

Im zugrundeliegenden Fall klagte eine 34jährige Mutter dreier Kinder gegen einen Leitenden Arzt einer Frauenklinik, der sie bei der Entbindung von ihrem dritten Kind auf ihren Wunsch hin durch eine Tubenligatur unfruchtbar gemacht hatte. Der Arzt hatte mit der Frau die Frage, ob eine Sterilisation bei der Geburt des dritten Kindes vorgenommen werden sollte, bereits bei den Schwangerschaftsuntersuchungen erörtert. Die Klägerin verlangte von dem Beklagten ein angemessenes Schmerzensgeld. Landgericht und Oberlandesgericht hatten die Klage abgewiesen, der Bundesgerichtshof hat den Eingriff für rechtmäßig erachtet und die Klage ebenfalls abgewiesen.

In der ausführlichen Begründung des BGH wird gesagt, da $\beta$ der Klägerin ein Schmerzensgeld nicht zustände, weil sie in die Sterilisation eingewilligt habe und diese Einwilligung wirksam sei. Der BGH ging davon aus, daß der ärztliche Eingriff in die körperliche Integrität tatbestandsmäßig eine Körperverletzung im Sinne von $\$ 823$ Abs. 1 BGB darstellte, für deren Rechtmäßigkeit es auf besondere Rechtfertigungsgründe ankomme. Ein Arzt, der eine 34jährige Frau und Mutter von 3 Kindern unfruchtbar macht, wenn sie das wünscht, weil sie keine weiteren Kinder haben will, handelt nicht rechtswidrig. Der BGH vertritt die Ansicht, daß die Beurteilung der Rechtmäßigkeit einer Sterilisation sich an dem allgemeinen Grundsatz zu orientieren habe, daß jeder selbst darüber bestimmen könne, ob er einen ärztlichen Eingriff an sich vornehmen lassen wolle, ein Grundsatz der nur dann eine Ausnahme erfahre, wenn der Eingriff trotz seiner Einwilligung gegen die guten Sitten verstoße. Nach Ansicht des BGH verletze die freiwillige Sterilisation in Fällen wie dem vorliegenden nicht die Grundvorstellungen von dem, was nach den herrschenden Anschauungen unseres Rechts- und Kulturkreises innerhalb der sozialen Gemeinschaft vom einzelnen an sittlichem Verhalten verlangt würde. In den weiteren Ausführungen geht der BGH darauf ein, daß nach der herrschenden Moralvorstellung weder Empfängnisverhütung als solche noch ärztliche Mithilfe dazu verwerflich sei. Im Gegenteil werde die freie Entscheidung für oder gegen eine Elternschaft als Möglichkeit zu einer humaneren Lebensführung verstanden.

Die sittlichen Werte von Ehe und Familie würden - jedenfalls nach den hier maßgebenden Moralvorstellungen - durch solche Entscheidungsfreiheit nicht angetastet. Der 
BGH weist aber weiter darauf hin, daß die Problematik der Zulässigkeit der freiwilligen Sterilisation nach wie vor umstritten sei. Die Lehrmeinungen der Kirchen würden sie in ihrem Grundsatz ablehnen, der Deutsche Ärztetag habe sich 1970 und 1976 in Entschließungen dafür ausgesprochen, die Sterilisation nur aus medizinisch-genetischen oder schwerwiegenden sozialen Gründen zuzulassen.

Es sei für die Beurteilung, inwieweit die Gesellschaftsordnung die mit der Sterilisation verbundene Verkürzung der Persönlichkeit des einzelnen hinzunehmen habe, der Entscheidungsfreiheit des Betroffenen, die auch das Risiko des „unrichtigen Gebrauchs“ zum Nachteil der eigenen Interessen mit umfasse, besonderes Gewicht einzuräumen. Der Bundesgerichtshof betont, wie bereits im Dohrn-Urteil, daß, solange es an einer verbindlichen Entscheidung des Gesetzgebers fehle, die Entscheidung dem Einzelfall vorbehalten bleiben müsse.

Obwohl der BGH sich nicht mit der strafrechtlichen, sondern mit der zivilrechtlichen Seite der freiwilligen Sterilisation auseinandersetzt, geht der Zivilsenat in seiner Entscheidung vom 29.6. 1976 davon aus, daß seit dem DohrnUrteil freiwillige Sterilisationen strafrechtlich nicht mehr verfolgt werden.

Zur zivilrechtlichen Seite des Falles wird weiter ausgeführt, daß für die Rechtmäßigkeit einer Sterilisation selbstverständlich die Einwilligung des Betroffenen vorliegen müsse, die Einwilligung müsse in Kenntnis ihrer vollen Tragweite erklärt worden sein. Obwohl eine schriftliche Einverständniserklärung der Klägerin nicht vorgelegen hatte, war der BGH der Meinung, daß ein mündlich erklärtes Einverständnis im vorliegenden Fall genügt habe, weil die Klägerin während vorausgegangener Schwangerschaftsuntersuchungen wiederholt den Wunsch nach einer Tubenligatur geäußert habe. Generell wird in der Urteilsbegründung vom BGH dem Arzt allerdings zur schriftlichen Fixierung der Einverständniserklärung geraten.

Mit diesem neuerlichen richtungweisenden Urteil von 1976 wird betont, daß der BGH keinesfalls in seiner Entscheidung sagen wolle, die freiwillige Sterilisation sei nunmehr ohne Einschränkung freizugeben. Der Arzt ist nach wie vor verpflichtet, in jedem Einzelfall zunächst genau zu prüfen, ob sein Tun rechtmäßig ist.

In den Kommentaren zu dieser Urteilsfindung des BGH wird darauf abgezielt, daß wiederum keine vollständige Klärung der Frage um die Zulässigkeit der freiwilligen Sterilisation durch den $\mathrm{BGH}$ herbeigeführt wurde. Als gesichert kann lediglich angenommen werden, daß Sterilisationen aus sozialen Gründen in weitgehendem Sinne nicht als sittenwidrig zu betrachten sind. Die Problematik der Gefälligkeitssterilisation hängt nach wie vor in der Luft.

Das gerichtliche Szenarium hat sich nach dem Dohrn-Urteil und dem BGH-Urteil von 1976 zur zivilrechtlichen Seite verlagert. Es konnte beobachtet werden, daß sich Klagen auf Schadensersatz und Schmerzensgeld aus Anlaß einer Sterilisation häuften, weiterhin waren fehlerhaft geführte Aufklärung bzw. Einwilligungsmängel Gegenstand von Prozessen.

Ein weiterer Punkt, der Anlaß zum Rechtsstreit geben kann, ist bei einer gegebenen Einverständniserklärung zur Sterilisation die Einwilligungsfähigkeit. Ihre Bedeutung ist zunächst einmal darin zu sehen, daß hier die unmittelbare Anwendung der für die Geschäftsfähigkeit geltenden Regeln des BGH ausgeschlossen sind. Statt auf die Volljährigkeit kommt es auf die konkrete „Einsichts- und Urteilsfähigkeit“ an. Diese ist nicht mit einer gesetzlichen Altersgrenze verknüpft, sondern damit, ob der zu Behandelnde im Einzelfall Art und Tragweite des bei ihm vorzunehmenden Eingriffs voll $\mathrm{zu}$ erfassen vermag. In Rechtskommentaren zum StGB wird teilweise ein Mindestalter von 25 Jahren vorausgesetzt, andererseits aber auch wieder auf die Volljährigkeitsgrenze abgehoben. Die Rechtsprechung hat in dieser Frage noch keine Stellung beziehen müssen, in einschlägigen zivilrechtlichen Urteilen wurde die Einwilligungsfähigkeit der zum Operationszeitpunkt zwischen 25 und 34 Jahren alten Betroffenen jeweils stillschweigend bejaht. Allein schon die Bandbreite der vertretenen Altersgrenzen schränkt ihre praktische Brauchbarkeit als Beurteilungshilfe ein. An die Aufklärung bei einer freiwilligen Sterilisation werden äußerst hohe Anforderungen gestellt, weil es sich um einen nicht dringlichen Eingriff handelt, dem dazu noch in der Regel nicht die Qualität einer Heilbehandlung, sondern eher einer kosmetischen Operation zukommt. Obwohl bereits im rechtsmedizinischen Schrifttum über die Aufklärungsrichtlinien berichtet wurde, sollen diese Punkte in unserem Zusammenhang nochmals dargestellt werden:

a) Es muß die Art der Behandlung genau erklärt werden.

b) Es müssen die mit dem Eingriff zwangsläufig verbundenen Folgen, insbesondere die dauernde unwiderrufliche Unfruchtbarkeit, Operationsnarben, aber auch psychische Entlastung von Schwangerschaftsfurcht erwähnt werden.

c) Es müssen etwaige unbeabsichtigte Nebenwirkungen und Risiken und zwar sowohl hinsichtlich möglicher Komplikationen bei der Durchführung des Eingriffs selbst (Narkoserisiko, Komplikationen des operativen Zugangsweges und der eigentlichen Sterilisationsmethode) als auch hinsichtlich potentieller körperlicher und psychischer Spätfolgen erwähnt werden.

d) Es muß auf die Gefahr von Sterilisationsversagern z. B. durch spontane Rekanalisation hingewiesen werden.

e) Es muß über etwaige alternative Methoden der Fruchtbarkeitsverhütung und ihre jeweilige kontrazeptive Sicherheit belehrt werden.

In dieser Übersicht ist zum Ausdruck gekommen, daß in der Praxis eine Fülle von Rechtsfragen vorliegt, die keinesfalls eindeutig erklärt sind. Der Gesetzgeber hat bislang zu diesem Problem keine eindeutige Stellungnahme abgegeben, eine endgültige Regelung ist zum gegenwärtigen Zeitpunkt auch noch nicht in Sicht. Vor wenigen Jahren stand sicherlich noch eine strafrechtliche Klärung der prinzipiellen Zulässigkeit von nicht indizierten Sterilisationsoperationen im Vordergrund, inzwischen haben sich die Gewichte jedoch verschoben. Haftungsfragen, Fragen der Unterhaltsleistung, Schmerzensgeldansprüche und ähnlich gelagerte zivilrechtliche Probleme sind in den Blickpunkt des Interesses getreten.

Nach unserer Meinung sollte aufgrund der diskutierten Probleme, die nach wie vor einer eindeutigen Lösung bedürfen, die Indikation zur Vasektomie aus Gründen der Gefälligkeit mit größter Zurückhaltung gestellt werden. Da keine Handhabe gegen den Arzt besteht, ihn zur Durchführung dieses Eingriffes zu zwingen, lassen sich durch Unterlassung dieser Sterilisationen eventuelle unangenehme Folgen vermeiden. 


\section{Literatur}

1 BGH, Arztrecht 16 (1981), 255, 266

2 BGH, MedR 1983, 101

3 BGH, NJW 1972, 520

4 BGH, NJW 1974, 1424

5 BGH, NJW 1976, 1790

6 BGH, NJW 1980, 1450

7 BGH, NJW 1981, 630

8 BGH, NJW 1981, 2002

9 BGH, NJW 1984, 2625

10 BGH, JZ 1964, 296

11 BGH, JZ 1965, 220

12 BGH, JZ 1980, 406

13 Böckle, F.: Ethische Aspekte der freiwilligen operativen Sterilisation, Stimmen der Zeit 1974, 755

14 Braun, W., W. Spann: Ein neues Urteil zur Frage der Zuverlässigkeit der freiwilligen Sterilisation, Geb Fra 37 (1977), 1

15 Brenner, G. (Hrsg.): Arzt und Recht, 119, Gustav-Fischer-Verlag, Stuttgart - New York, 1983

16 Brenner, G.: Rechtliche Zulässigkeit der Sterilisation (dauernde Empfängnisverhütung), Geb Fra 42 (1982), 226

17 Deutsch, E. (Hrsg.): Arztrecht und Arzneimittelrecht, 160, Springer Verlag, Berlin - Heidelberg - New York, 1983

18 Erbgesundheitsgesetz (ErbGesG) vom 14.7. 1933 (RGB\ I. S. 529) i. d. F. vom 26.6. 1935 (RGBl I, S. 773)

19 Eser, A., H.-G. Koch: Aktuelle Rechtsprobleme der Sterilisation, Gynäkologe 15 (1982), 62

20 Hanack, E.-W.: Die Sterilisation aus sozialer Indikation, JZ 1964, 299

${ }^{21}$ Hanack, E.-W., H.-D. Hiersche: Der Schwangerschaftsabbruch: Rechtsfragen aus der Praxis, Arch Gyn 228 (1979), 331

22 Hellmann, R.: Die Sterilisierung als legitime ärztliche Aufgabe, Med. Welt 21 (1970), 41

23 Hild, F.: Haftpflichtfragen nach Vasektomie, Urologe B 18 (1978), 149

24 Hirsch. H. A.: Schwangerschaften nach fehlgeschlagener Tubensterilisation, Deutsches Ärzteblatt 78 (1981), 1669

25 Hoerster, N.: Grundsätzliches zur Strafwürdigkeit der Gefälligkeitssterilisation, JZ 1971, 123

26 Horn, E.: Strafbarkeit der Zwangssterilisation, ZRP 1983, 265

${ }_{27}$ Kaiser, G.: Eugenik und Kriminalwissenschaft heute, NJW 1969, 538

${ }^{28}$ Koffka, E.: Wie soll die freiwillige Sterilisation künftig gesetzlich geregelt werden? In: Heusinger-Festschrift, 355, Beck-Verlag, München, 1968
${ }^{29} \mathrm{Kunz}, \mathrm{K} .-\mathrm{L} . \mathrm{:}$ Die strafrechtliche Problematik der freiwilligen Sterilisation, JZ 1982, 788

30 Laufs, A.: Arztrecht im Wandel, NJW 1977, 1081

31 LG Berlin, FamRZ 1971, 668

32 LG Düsseldorf, FamRZ 1981, 95

33 OLG Bamberg, NJW 1978, 1965

34 OLG Braunschweig, NJW 1980, 643

35 OLG Celle, NJW 1963, 407

36 OLG Celle, MedR 1984, 233

37 OLG Düsseldorf, VersR 1975, 432

38 OLG Düsseldorf, NJW 1975, 595

39 OLG Frankfurt, MedR 1983, 70

${ }^{40}$ Petersen, P. (Hrsg.): Sterilisation. Beratung, Operation, Recht. Thieme-Verlag, Stuttgart - New York, 1981

41 Rieger, H.-J.: Rechtmäßigkeit der freiwilligen Sterilisation, DMW 17 (1977), 666

42 Rieger, H.-J.: Sterilisation aus sozialer Indikation, DMW (1973), 1781

${ }^{43}$ Röhmel, K.: Das Problem der Strafwürdigkeit der freiwilligen Sterilisation, JA 1977, 183

44 Roxin, C.: Entwicklung und gesetzliche Regelung des Schwangerschaftsabbruches, JA 1981, 226

45 Roxin, C.: Verwerflichkeit und Sittenwidrigkeit als unrechtsbegründende Merkmale im Strafrecht, JuS 1964, 373, u. ders., Nieders. Ärzteblatt 1965, 165

46 Schirren, C.: Vasektomie - Pro und Kontra, MMW 122 (1980), 553

47 Schlund, G. H.: Rechtsfragen bei der Vasektomie, Urologe B 18 (1978), 152

48 Schmidt, E.: Das Sterilisationsproblem nach dem in der Bundesrepublik geltenden Strafrecht, JZ 1951, 65

49 Spann, W.: Rechtsgrundlagen der operativen Sterilisation beim Mann und bei der Frau in der Bundesrepublik Deutschland, Geb Fra 35 (1975), 501

50 Spann, W.: Zur besonderen Problematik der Sterilisation aus sozialer Indikation und der Gefälligkeitssterilisation, Geb Fra 36 (1976), 197

51 Wagenknecht, L. V.: Operative Möglichkeiten der Refertilisierung beim Mann, MMW 122 (1980), 549

52 Wulfhorst, T.: Wäre eine Strafbarkeit der freiwilligen Sterilisation verfassungswidrig? NJW 1967, 649

Dr. V. Müller-Mattheis

Urologische Universitätsklinik

Moorenstr. 5

4000 Düsseldorf

1. Die Verfasser haben ausgeführt, daß nach der Rechtsprechung grundsätzlich jede ärztliche Maßnahme, die die Integrität des Körpers berührt, tatbestandsmäßig eine Körperverletzung darstellt. Bei der Gefälligkeitsvasektomie ist jedoch umstritten, ob der Tatbestand der Körperverletzung erfüllt ist; nach der Rechtsprechung ist das nicht der Fall, während nach Ansicht weiter Teile des Schrifttums eine Körperverletzung tatbestandlich vorliegen soll: Nach dieser in der Literatur vertretenen Meinung ist die Gefälligkeitsvasektomie, wenn sie nicht als Nebenfolge eines bereits anderweitig medizinisch indizierten Eingriffs als normale Heilbehandlung durchgeführt wird, tatbestandsmäßig eine schwere Körperverletzung

(\$§ 223, 224, 225 StGB). Sie bedarf der Rechtfertigung durch Einwilligung und ist nur beim Vorliegen einer bestimmten Indikation nicht sittenwidrig ( $\$ 226 \mathrm{a}$ StGB). Anderer Ansicht ist jedoch der Bundesgerichtshof: Der Bundesgerichtshof hält die freiwillige Sterilisation wegen Fehlens einer besonderen Strafdrohung schon gar nicht erst für tatbestandsmäßig und daher auch nicht für indikationsbedürftig (BGHSt 20, 81).

a) Früher war das anders: Kastration und Sterilisation wurden wie jeder andere ärztliche Eingriff auch als Körperverletzung angesehen. Durch die Einführung von $\$ 14$ ErbGesG vom 26. 6. 1935 und durch $\S 226$ b von 1943 wurden beide Eingriffe tatbestandlich gesondert geregelt und waren nur unter bestimmten Voraussetzungen zulässig. § 226b StGB wurde 1946 aufgehoben. Aus der Normierung und späteren Streichung des $§ 226 \mathrm{~b}$ StGB schloß der Bundesgerichtshof, daß freiwillige Sterilisationen nicht mehr tatbestandsmäßig vom Strafgesetzbuch erfaßt seien und auch nicht sittenwidrig sein könnten, denn anderenfalls hätte der Gesetzgeber die Vor- 
schrift nicht aufheben dürfen (BGHSt 20, 81). Diese Argumentation des Bundesgerichtshofs im sogenannten Dohrn-Urteil ist unabhängig von ihrem Ergebnis rechtsdogmatisch wohl nicht haltbar, weil eigentlich der frühere Zustand vor Schaffung des $\$ 226 \mathrm{~b}$ StGB durch die Aufhebung der Vorschrift wiederhergestellt wurde, d.h., die Gefälligkeitsvasektomie erfüllt den Tatbestand der Körperverletzung. Konkret führt die Rechtsprechung des Bundesgerichtshofs unabhängig davon, ob sie nun haltbar ist oder nicht, und unabhängig davon, ob der Tatbestand der Körperverletzung vorliegt oder nicht, zu folgendem Ergebnis: Ein Strafverfolgungsrisiko ist praktisch auszuschließen, wenn die freiwillige Sterilisation durch die Einwilligung des Betroffenen gedeckt ist und sie kunstgerecht durchgeführt wird (so auch: Eser, in: Schönke/Schröder, StGB-Kommentar, 22. Aufl., München 1985, § 223 Anm. 62. m.w.N.).

b) Die Einwilligung bei der Gefälligkeitsvasektomie, die sich auf keine rechtfertigende Indikation berufen kann und die nur durchgeführt wird, um jegliches Schwangerschaftsrisiko auszuschalten, ist insbesondere nicht wegen $\$ 226$ a StGB sittenwidrig und damit nichtig, denn die Sittenwidrigkeit läßt sich angesichts der kontrovers geführten Diskussion in der juristischen Literatur nicht begründen. Etwas anderes ergibt sich auch nicht aus $\$ 6$ BOÄ, der nur die medizinische, eugenische und schwerwiegende soziale Indikation zuläßt, die Gefälligkeitsvasektomie also nicht erwähnt. Für die Beurteilung der Voraussetzungen des $\$ 226 \mathrm{a}$ StGB ist die Standesethik zwar durchaus von Bedeutung, jedoch ist sie kein letztverbindlicher Maßstab.

2) Da nach der Rechtsprechung die Gefälligkeitsvasektomie an sich ohne Strafverfolgungsrisiko durchgeführt werden kann, kommt es wie bei jedem anderen ärztlichen Eingriff auf die wirksam erteilte Einwilligung des Betroffenen an. Ist sie nicht wirksam erteilt, so liegt keine freiwillige Sterilisation vor, und der Arzt hat eine rechtswidrige Körperverletzung ( $\$ 223$ StGB, evtl. auch nach § 225 StGB) begangen.

a) Eine wirksame Einwilligung setzt auf jeden Fall zunächst eine ordnungsgemäße ärztliche Aufklärung voraus. Ohne Aufklärung ist die Einwilligung in der Regel rechtlich unbeachtlich (es sei denn, der Betreffende ist schon voll aufgeklärt, etwa weil er selbst Facharzt ist). Die Notwendigkeit der Aufklärung ergibt sich nicht nur als therapeutische Maßnahme aus der lex artis oder dem Behandlungsvertrag, sondern vor allem aus dem verfassungsrechtlich garantierten Persönlichkeitsrecht des Betroffenen, das die Selbstbestimmung des einzelnen umfassend gewährleistet. Die Selbstbestimmungsaufklärung soll ihm Art, Bedeutung und Tragweite des Eingriffs deutlich machen (beim routinemäßigen Heileingriff, den die Gefälligkeitsvasektomie auf keinen Fall darstellt, wenigstens in den Grundzügen im großen und ganzen, um ihm so eine $\mathrm{Ab}$ schätzung des Für und Wider des Eingriffs zu ermöglichen). Die Aufklärung bezieht sich auf alle Umstände, die für die Entscheidung eines „verständigen “ Patienten ins Gewicht fallen können. Es kommt auf alle Umstände des Einzelfalles an - vor allem ist die Aufklärungspflicht um so weitergehend, je leichter aufschiebbar und je weniger geboten die ärztliche Maßnahme (wie bei der Gefälligkeitsvasektomie) ist. Bei der Gefälligkeitsvasektomie ist eine weitestgehende(!) Aufklärungspflicht anzunehmen. Darin liegt das strafrechtliche Risiko für den Arzt. Aufzuklären hat grundsätzlich der den Eingriff eigenverantwortlich durchführende Arzt, wobei aber der Klinikchef in gewissen Grenzen überwachungspflichtig ist. Aufgeklärt werden muß zumindest über folgendes: Art, Ziel und Verlauf der Behandlung sowie über Alternativen dazu, außerdem über die Folgen des Eingriffs und des damit verbundenen Risikos und über die Diagnose, jedoch nur ganz ausnahmsweise über die Kosten des Eingriffs. Die Aufklärung muß rechtzeitig erfolgen. Wichtig ist, daß selbst ein nachweisbarer Aufklärungsmangel nur zur Strafbarkeit des Arztes führt, wenn bei ordnungsgemäßer Aufklärung die Einwilligung nicht erteilt worden wäre. Anders als nach den zivilprozessualen Beweislastregeln ist dem Arzt im Strafrecht die Kausalität des Aufklärungsmangels für die sonst verweigerte Einwilligung nachzuweisen. Nur der Patient selbst ist im übrigen einwilligungsberechtigt, denn es geht beim ärztlichen Eingriff um ein höchstpersönliches Rechtsgut, um die Gesundheit (für die Beurteilung der Wirksamkeit der Einwilligung sind nicht die zivilrechtliche Geschäftsfähigkeit oder die strafrechtliche Schuldfähigkeit von Bedeutung, sondern nur die natürliche Einsichts- und Urteilsfähigkeit). Fehlt dem Patienten die eigene Entscheidungsfähigkeit, so muß der gesetzliche Vertreter sein Einverständnis wirksam erklären (bei der Gefälligkeitsvasektomie wird es im übrigen auf die mutmaßliche Einwilligung kaum ankommen). Die Einwilligung ist nur wirksam erteilt, wenn sie frei von Willensmängeln ist, also nicht bei arglistiger Täuschung, Drohung, Irrtum.

b) Die Gefälligkeitsvasektomie enthält im Vergleich zu anderen ärztlichen Eingriffen an sich zwar kein gesteigertes Risiko für den Arzt; das strafrechtlich kaum noch kalkulierbare Risiko für den Arzt liegt aber darin, daß sich der Betroffene nach Durchführung des Eingriffs anders besinnt und er dem Arzt eine Verletzung der Aufklärungspflicht vorwirft. Die Aufklärungspflicht geht bei der Gefälligkeitsvasektomie so weit, daß der Arzt bei seiner Verteidigung in sehr ernsthafte Schwierigkeiten geraten kann.

K. Wasserburg (Mainz) 